\title{
TOTAL TRAVEL TIME ANALYSIS FOR STUDENTS IN A METROPOLITAN AREA: A STUDY FROM INDIA
}

\author{
Krishnapriya Molath Gopi ${ }^{1}$, Soosan George Tanenlimalil ${ }^{2}$, Bybin Paul ${ }^{3}$ \\ 1,3 Department of Civil Engineering, Mar Athanasius College of Engineering, Kerala, India \\ ${ }^{2}$ Department of Civil Engineering, Saintgits College of Engineering, Kerala, India
}

Received 25 July 2019; accepted 2 September 2019

\begin{abstract}
Efficient transportation system management is possible only through managing travel needs of commuters, using travel demand models. The extend by which a commuter need to travel for accomplishing his/her daily needs is here represented by the total travel time. Total travel time is one of the activity-travel behaviour which is least considered by transportation researchers. Travel demand studies often focus the workers, but give little attention to the students. In a developing country like India, students also contribute a major share in morning and evening peak hour traffic. This study presents the analysis of total travel time for the student community incorporating the socio-demographic features using activity based modelling approach. Preliminary analysis gives details on daily activity-travel pattern, mode choice preferences and other particulars of students in the study area. Statistical models are developed and simulation of choice probabilites is also done for understanding the factors affecting total travel time behaviour, for students in a usual working day.
\end{abstract}

Keywords: activity based modelling, total travel time, disaggregate model, students, developing country.

\section{Introduction}

Activity based approach in travel demand modelling is the latest and accurate travel demand modelling approach which works on the basic assumption that travel is a derived demand, which is derived from the need of individuals to perform an activity. The fundamental theories behind activity based approach were developed during 1970s (Hägerstrand, 1970; Chapin, 1974; Cullen and Godson, 1975). An individual have to perform different activities like work, education, shopping, recreation etc. to fulfill his/her day-to-day needs. There will be in-home and out-home activities, among which the individuals need to travel for performing out-home activities. The extent to which each commuter needs to travel can be effectively represented by the travel time for that particular activity. According to activity the total population can be divided in to segments like workers, students etc. Most of the travel studies focus on workers, but students are usually neglected. In a developing country like India, the share of student category is also very big next to workers and therefore it is necessary to analyse different travel behaviour elements related to students, for deriving better solutions for transportation problems.

${ }^{1}$ Corresponding author: krishnapriyamg@mace.ac.in 
An appropriate representation of all sub population will definitely improve the regional travel demand models. Among these sub population workers are given importance in most of the transportation researches and the presence of students are mostly absent. Only a few researches have taken the students into consideration and its exploratory analysis itself gives out that the students behave differently compared to the general population (Khattak et al., 2011). Mode choice behaviour of students is modelled in Germany and Canada, using multinomial logit modelling (MNL) method (Müller et al., 2008; Whalen et al., 2013; Mitra and Buliung, 2014).

Accessibility indicators and travel time can be used as the variables that represent ones friction to travel for participating different activities (Frank et al., 2008). Travel time for different modes is also compared in order to find the relationships with environmental accessibility (Salonen and Toivonen, 2013). Total travel time can also be considered as an urban mobility performance measure to address different transportation congestion problems (Lomax and Schrank, 2010). Different travel demand modelling studies conducted in India for all segments of population is also an important element of discussion. The exploratory and statistical analysis of activity-travel behaviour of nonworkers in Bangalore city were studied. Analysis of activity participation behaviour, trip chaining, mode choice behaviour, etc. was done using MNL modelling method (Manoj and Verma, 2015). Mode choice analysis of workers in Tiruvanthapuram City, Kerala, was also done using MNL modelling method. Its results shows lower age group prefers two-wheeler and higher age group prefer car compared to bus. Female commuters prefer public vehicle and male commuters prefer private vehicles more. Other parameters like income, vehicle ownership, distance, time/distance and cost/distance also have significant effect on the mode choice decision (Ashalatha et al., 2013). Mode choice decision of workers in Chennai city is also modelled. Study include alternate behavioural framework such as random utility minimisaiton, random regret minimisation etc. in addition to most widely used random utility maximisation rule for mode choice decision. Policy results are also evaluated for comparing the performance of all these rules used for selecting the mode choice (Parthan and Srinivasan, 2013). Activity travel pattern analysis for workers in Calicut City, Kerala is also performed using mutinomial logit modelling method (Sreela and Anjaneyulu, 2018).

However, the attempts to develop models to represent the travel behaviour of students are comparatively less, especially for developing countries. Major studies contribute into mode choice or school choice behaviour of students. Total travel time is also an important variable to be considered in choice modelling, as it is measure of extend of travel as well as a choice set over which total travel time reducing measures can be explored. In the present study the total travel time behaviour of students is analysed using disaggregate modelling method. The objectives of the study is to model the total travel time behaviour of students to identify the variables that have significant effect on it and also to perform simulation of choice probabilities on potential variables, whose variation can influence the total travel time behaviour of students. 


\section{Data Analysis and Modelling Framework}

Total travel time (TT) of an individual is here calculated as the total travel time taken for a single tour for the primary activity. For students education is their primary activity and illustration of total travel time (TT) calculated for an education tour is shown in Fig. 1. The travel time taken by a student to travel from home to school/college and back from school/college to home is considered as the total travel time. Students who are not permanently residing at their home are not considered for modelling. In the case of public/school bus the total travel time includes time for accessing bus stop, waiting time and time to reach school/college from bus stop. Based on the total travel time, the tours are classified into three, they are short tours (TT1) in which $\mathrm{TT} \leq 30$ minutes, medium tours (TT2) in which TT between $31-60$ minutes and long tours (TT3) in which $\mathrm{TT} \geq 61$ minutes; as per referring similar studies (van Exel and Rietveld, 2010) and opinion from transportation experts.

$\mathrm{TT}_{\mathrm{HE}}=30$ mins.

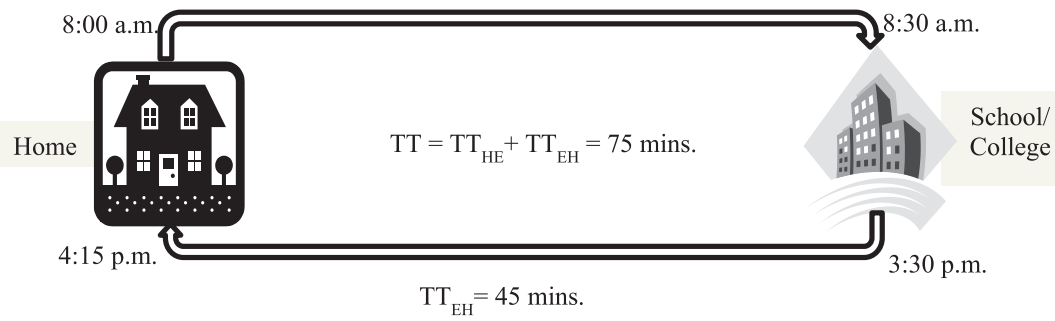

Fig. 1.

Total Travel Time for a Tour for Education

\subsection{Study Area and Data Collection}

Kochi Municipal Corporation, Kerala is the study area selected for the present study. Kochi is the commercial, industrial and financial capital of Kerala and the most densely populated corporation in the state, with a population density of 6340 persons/ $\mathrm{km}^{2}$. As per 2011 Census, population of Kochi is 602,046 with 296,949 males and 305,097 females. Literacy rate of KMC is $97.36 \%$, which is very high compared to any other major cities in India. Kochi Municipal Corporation spreads over an area of $94.88 \mathrm{~km}^{2}$ and it is well connected by road, rail, air and water modes. Total road length for Kochi city is $614 \mathrm{~km}$ and the existing road density is $6.47 \mathrm{~km} / \mathrm{sq} . \mathrm{km}$. The narrow roads and improper planning of city area have made it one of the cities with worst traffic conditions within India. Kochi is the potential target of central and state government authorities to implement sustainable development strategies to mitigate the existing traffic problems. Kochi city is selected as 5th among the 20 major cities in the first round selection under Smart City Mission by Ministry of Urban development, Government of India. It is also considered under Kerala Sustainable Urban Development Project (KUDP) by Kerala Government.

Primary and secondary data is used for modelling the total travel time behaviour of 
students. Direct household interview method is adopted for primary data collection and total 2989 household details are there in the final database for analysis. An official consent letter was issued for data collection by Kochi Mayor, which have successfully increased the response rate of survey to $96.67 \%$ (Gopi et al., 2018). Secondary data is collected from reports of government bodies as well as from former researches within the study area.

\subsection{Multinomial Logit (MNL) Models}

Multinomial logit model has been widely used for choice modelling, which gives the choice probabilities of each alternative as a function of the systematic portion of the utility of all the alternatives (Koppelman and Bhat, 2006). The basic form of MNL model for probability of choosing alternative $\mathrm{j}$ is shown in Equation (1).

$\operatorname{Pr}($ Choice $\mathrm{j})=\frac{\exp \left(\beta_{\mathrm{j}} \mathrm{x}_{\mathrm{ji}}\right)}{\sum_{\mathrm{m}=1}^{j} \exp \left(\beta_{\mathrm{m}} x_{\mathrm{mi}}\right)}, \mathrm{j}=0, \ldots, \mathrm{J}$,

Where ' $i$ ' indicates the observation or individual and ' $j$ ' and ' $m$ ' indicates the choices. ' $\beta$ ' represents the coefficient of the variable ' $x$ ' considered. The decision of choosing an alternative is based on utility maximisation rule. It can be stated as alternative, ' $i$ ', is chosen among a set of alternatives, if and only if the utility of alternative, ' $i$ ', is greater than or equal to the utility of all alternatives, ' $j$ ', in the choice set, $C$. This can be expressed mathematically as shown in Equation (2):

If $\mathrm{U}\left(\mathrm{X}_{\mathrm{i}}, \mathrm{S}_{\mathrm{t}}\right) \geq \mathrm{U}\left(\mathrm{X}_{\mathrm{j}}, \mathrm{S}_{\mathrm{t}}\right) \forall \mathrm{j} \Rightarrow \mathrm{i}>\mathrm{j} \forall \mathrm{j} \in \mathrm{C}$

where $U()$ is the mathematical utility function, $\mathrm{X} i, X j$ are vectors of attributes describing alternatives $i$ and $j$, respectively, $S t$ is a vector of characteristics describing individual $t$ that influence his /her preferences among alternatives, $i>j$ means the alternative to the left is preferred to the alternative to the right, and $\forall j$ means all the cases, $j$, in the choice set. That is, if the utility of alternative $i$ is greater than or equal to the utility of all alternatives, $j$; alternative $i$ will be preferred and chosen from the set of alternatives, C. In this study MNL modelling is used for total travel time analysis.

\subsection{Model Estimation}

An estimation method which scores different estimation results in terms of how well they identify the chosen alternative is accomplished by using maximum likelihood estimation method. The procedure for maximum likelihood estimation involves two important steps: 1) developing a joint probability density function of the observed sample, called the likelihood function, and 2) estimating parameter values which maximize the likelihood function (Hensher et al., 2005). The likelihood function for a sample of ' $T$ ' individuals, each with ' $J$ ' alternatives is defined using the mathematical Equation (3):

$L(\beta)=\prod_{\forall t \in T} \prod_{\forall j \in J}\left(P_{j t}(\beta)\right)^{\delta_{j t}}$

Where $\delta_{j t}=1$ is chosen indicator $(=1$ if $j$ is chosen by individual $t$ and 0 otherwise) and $P_{j t}$ is the probability that individual $t$ chooses alternative $j$. Logarithm of the likelihood function is considered for simplifying the calculations as shown in Equation 4.

Log likelihood $=\operatorname{LL}(\beta)=\log (\mathrm{L}(\beta))$

The values of parameters that maximize likelihood function are obtained by finding the first derivative of likelihood function and equating it to zero. The models are calibrated using two third of the total data and one third is used for validation. 


\subsection{Measures of Goodness of Fit}

After calibration of the model it is tested for accuracy using goodness of fit measures. Goodness of fit measures is calculated for each parameter as well as for overall model. Wald statistics or P-value (significance value) is calculated for finding the significance of each parameter. The coefficient estimated is considered to be significant at $90 \%$ of confidence level or more when the absolute value of corresponding Wald statistics is 1.64 or more. Similar to higher value of likelihood function gives the better model, so as likelihood ratio index (rho-squared value) and adjusted rho-squared value. It is similar to R-squared value similar to regression analysis. The value should come in the range of $0-1$. Rho-square value and adjusted rho square value with respect to constant model is calculated as shown in Equation (5) and Equation (6).

Rho - square value with repsect to constants $=\rho_{c}^{2}=1-\frac{L L(\hat{\beta})}{L L(c)}$

Adjusted rho - square value w.r. t constants $=\bar{\rho}_{c}^{2}=1-\frac{\mathrm{LL}(\tilde{\beta})-\mathrm{K}}{\mathrm{LL}(\mathrm{c})-\mathrm{K}_{\mathrm{ms}}}$

Where $\operatorname{LL}(\widehat{\beta})$ is log likelihood value of the model calibrated, LL(c) is log likelihood value of constant only model calibrated, $\mathrm{K}$ is number of degrees of freedom (parameters) and $\mathrm{K}_{\mathrm{ms}}$ is number of degrees of freedom in constant only model. The likelihood ratio test can be used in the same way as F-test in the regression models. It is approximately equal to the chi-square value of the model. It is calculated as shown in Equation (7):

Likelihood ratio $=-2[\operatorname{LL}(\mathrm{c})-\operatorname{LL}(\widehat{\beta})]$

Percentage correctly predicted is also used to find the goodness of fit of the calibrated model. In the present study modelling is done using the software package NLOGIT 6 , by coding the utility equation for each alternative (Greene, 2016).

\subsection{Post Estimation Analysis}

After developing the final model post estimation analysis are done in order to find the potential policy variables. In this study the post estimation techniques like calculation of elasticity and simulation of choice probability is done. Simulation of choice probability gives the variation of probability of choosing an alternative for a particular variation in the value of an attribute. Whereas, elasticity are calculated for the behavioural interpretation of an attribute over the alternative, which is not possible to explain using the sign of estimated parameter. Elasticity is a unit less measure that can describe the relationship between the percentage changes in probability of quantity demanded with respect to percentage change in some independent variable (Hensher et al., 2005). The direct elasticities for MNL model can be mathematically explained as the elasticity of probability of alternative $i$ for decision maker $t$, with respect to a change in the $k^{\text {th }}$ attribute of the $i^{\text {th }}$ alternative i.e. $X_{i k t}$, as observed by decision maker $t$ which can be calculated as shown in Equation (8).

$$
E_{X_{i k t}}^{P_{i t}}=\frac{\partial P_{i t}}{\partial X_{i k t}} * \frac{X_{i k t}}{P_{i t}}
$$




\section{Data Structure}

Total 2527 home based education samples are available for total travel time modelling. In which 1685 samples are used for model calibration and 842 samples are used for model validation. Personal, household, activity-travel and residential location variables are used for model calibration. Percentage distribution of tours with respect to total travel time and distribution of age for the students is given in Fig. 2 and Fig. 3. The other categorical variables considered for modelling and its details are given in Table 1.

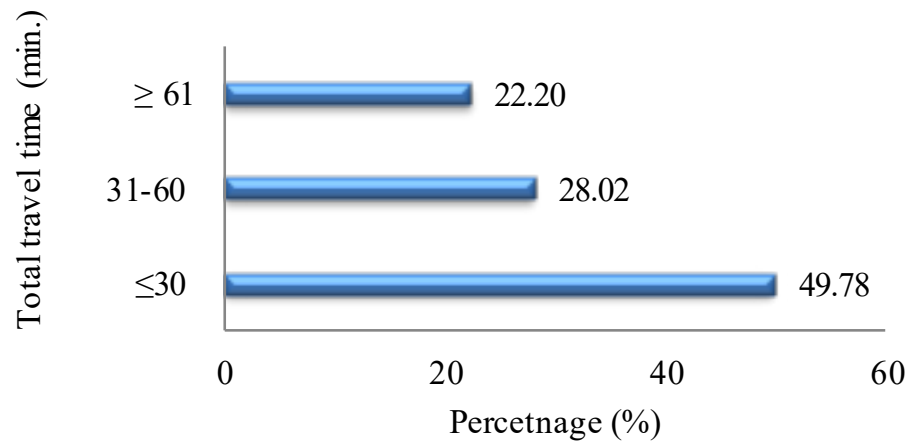

Fig. 2.

Percentage Distribution of Tours by Total Travel Time

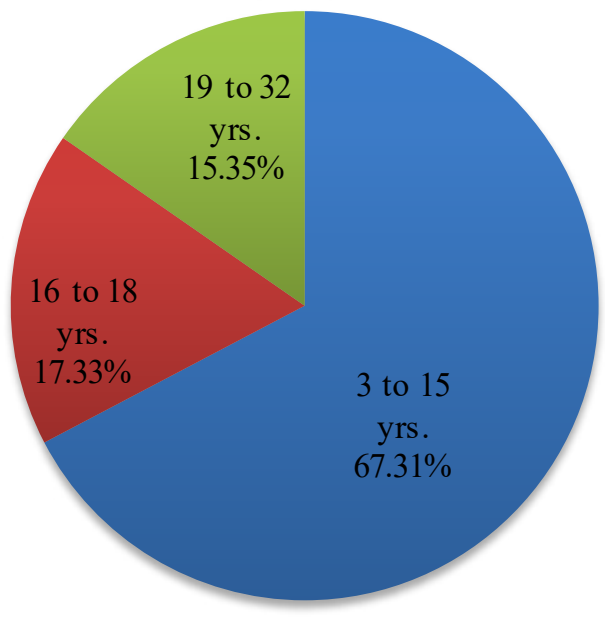

Fig. 3.

Percentage Distribution of Students by Age 


\section{Table 1}

Categorical Variables and Coding in Total Travel Time Model

\begin{tabular}{|c|c|c|c|}
\hline Variables & Coding & $\mathbf{N}$ & $\begin{array}{c}\text { Marginal } \\
\text { Percentage (\%) }\end{array}$ \\
\hline \multicolumn{4}{|l|}{ Gender } \\
\hline Male & GENM & 1273 & 50.38 \\
\hline Female & GENF & 1254 & 49.62 \\
\hline Driving License ownership & DLN & & \\
\hline Yes & 1 & 229 & 9.06 \\
\hline No & 0 & 2298 & 90.94 \\
\hline \multicolumn{4}{|l|}{ Education level } \\
\hline School level & ED1 & 1699 & 67.23 \\
\hline Higher secondary and more & ED2 & 828 & 32.77 \\
\hline \multicolumn{4}{|l|}{ Activity pattern } \\
\hline HEH (Simple) & $\mathrm{AP} 1$ & 2499 & 98.89 \\
\hline $\mathrm{HEH}+($ Complex $)$ & AP2 & 28 & 1.11 \\
\hline \multicolumn{4}{|l|}{ Mode used } \\
\hline Walk/ Bicycle & WCY & 661 & 26.16 \\
\hline Public vehicle & $\mathrm{PB}$ & 1656 & 65.53 \\
\hline Private Vehicle & PR & 210 & 8.31 \\
\hline \multicolumn{4}{|c|}{ Population Density (persons/hector) } \\
\hline$<25$ & PPD1 & 212 & 8.39 \\
\hline 25 to 50 & PPD2 & 1356 & 53.66 \\
\hline 50 to 100 & PPD3 & 743 & 29.40 \\
\hline$>100$ & PPD4 & 216 & 8.55 \\
\hline $\mathrm{N}$ & \multicolumn{3}{|c|}{2527} \\
\hline
\end{tabular}

In the total sample $50.38 \%$ are male and $49.62 \%$ are female. Only $9.06 \%$ of them have driving license ownership and almost $98 \%$ of them have simple activity pattern for education. Mode used and population density of the place of residence is also considered for modelling. The other variables are considered as explanatory variables and the coding, minimum value, maximum value, average and standard deviation of each variable is given in Table 2.

\section{Table 2}

Explanatory Variables and Coding in Total Travel Time Model

\begin{tabular}{|l|c|c|c|c|c|}
\hline Variables & Coding & Mean & $\begin{array}{c}\text { Minimum } \\
\text { value }\end{array}$ & $\begin{array}{c}\text { Maximum } \\
\text { value }\end{array}$ & $\begin{array}{c}\text { Standard } \\
\text { deviation }\end{array}$ \\
\hline Travel distance $(\mathrm{km})$ & TD & 9.58 & 0.10 & 60 & 8.58 \\
\hline Distance to nearest bus stop $(\mathrm{km})$ & DBS & 0.547 & 0.02 & 2.00 & 0.33 \\
\hline
\end{tabular}

\section{Results and Discussion}

Medium tour (TT2) is considered as the base alternative in total travel time model.
Significant parameter estimates, goodness of fit measures and validation results of total travel time model for students is given in Table 3. The value of Wald statistics and $95 \%$ 
confidence interval limits for the estimated parameters is also given in the table. Travel distance is one of the most influencing factors and as travel distance increases students have more probability to perform long tours and as travel distance decreases students have more probability to perform short tours. Choice of travel mode for education also has influence on total travel time. Students who prefer private mode, walk or bicycle usually perform short tours and students who choose public bus or school bus always took long time to travel for education. Travel time by public bus and school/college bus is comparatively very high even in cases where the travel distance is less.

Table 3

Parameters Estimated for Total Travel Time Model for Students

\begin{tabular}{|c|c|c|c|c|}
\hline Variables & Coefficient & Wald Statistics & \multicolumn{2}{|c|}{$95 \%$ confidence interval limits } \\
\hline \multicolumn{5}{|c|}{ Total travel time $\leq 30$ minutes (short tours) } \\
\hline Constant & 3.482 & 6.489 & 2.430 & 4.533 \\
\hline TD & $-0.583^{* * *}$ & -15.604 & -0.656 & -0.509 \\
\hline PR & $1.553^{* * *}$ & 3.963 & 0.785 & 2.321 \\
\hline WCY & $1.475^{* * *}$ & 5.633 & 0.962 & 1.988 \\
\hline GENM & $-0.328^{*}$ & -1.832 & -0.680 & 0.023 \\
\hline PPD1 & $0.565^{*}$ & 1.654 & -0.105 & 1.234 \\
\hline PPD4 & $-0.519^{*}$ & -1.748 & -1.101 & 0.063 \\
\hline DBS & $-0.712^{* *}$ & -2.499 & -1.271 & -0.154 \\
\hline \multicolumn{5}{|c|}{ Total travel time $\geq 61$ minutes (long tours) } \\
\hline Constant & -4.135 & -5.092 & -5.726 & -2.543 \\
\hline TD & $0.234^{* * *}$ & 12.708 & 0.198 & 0.271 \\
\hline GENM & $-0.342^{*}$ & -1.890 & -0.696 & 0.013 \\
\hline DBS & $0.439^{*}$ & 1.654 & -0.081 & 0.959 \\
\hline \multicolumn{5}{|c|}{ Goodness of fit measures } \\
\hline \multicolumn{4}{|c|}{ Log likelihood for constant only model } & -1752.861 \\
\hline \multicolumn{4}{|c|}{ Log likelihood at convergence } & -824.1151 \\
\hline \multicolumn{4}{|c|}{ Likelihood ratio /Chi square value (P-value) } & $1857.491(0.000)$ \\
\hline \multicolumn{4}{|c|}{ Rho-squared value } & 0.5298 \\
\hline \multicolumn{4}{|c|}{ Adjusted rho-squared value } & 0.5263 \\
\hline \multicolumn{4}{|c|}{ Percentage correctly predicted } & 79.407 \\
\hline \multicolumn{4}{|l|}{$\mathrm{N}$} & 1685 \\
\hline \multicolumn{4}{|c|}{ Validation: Percentage correctly predicted } & 73.63 \\
\hline \multicolumn{5}{|c|}{${ }^{* * *},{ }^{* *},{ }^{*}==>$ Significance at $1 \%, 5 \%, 10 \%$ level } \\
\hline
\end{tabular}

Gender of the students is also influencing the total travel time choice for education. Male students more prefer medium tours for education compared to short and long tours. Students coming from low population density region prefer short tours, whereas students from high population density region significantly least prefer short tours. Accessibility to nearest bus stop from place of residence is another factor that influence the total travel time. As accessibility to nearest bus stop decreases students have more tendencies to perform long tours and least tendency to perform short tours. Chi-square value of the final model has $\mathrm{P}$-value 0.000 , from which it is evident that the calibrated model is significantly different from the constant only model. Rho-squared and 
adjusted rho-squared value are reasonably good value and the percentage correctly predicted is also high i.e., $79.407 \%$. Model is also validated using $1 / 3 \mathrm{rd}$ of the total data and percentage correctly predicted value obtained is $73.63 \%$, whose variation is within $10 \%$ with that of calibrated data. So the model can be considered as a reasonably good model for total travel time analysis of students.
Point elasticities and cross elasticities are calculated for the exploratory variables and given in Table 4. The calculated elasticities shows that an increase in travel distance by unit percentage can decrease the probability of choosing short tour by $4.65 \%$ and increase the probability of choosing long tour by $1.79 \%$. Distance to nearest bus stop has point elasticities for short tour and long tour are -0.336 and 0.198 respectively.

\section{Table 4}

Elasticities of Explanatory Variables in Total Travel Time Model for Students

\begin{tabular}{|c|c|c|}
\hline Alternatives & Travel distance (TD) & $\begin{array}{c}\text { Distance to nearest bus stop } \\
\text { (DBS) }\end{array}$ \\
\hline \multicolumn{3}{|c|}{ Point elasticities } \\
\hline Short tour & -4.6500 & -0.3361 \\
\hline Medium tour & - & - \\
\hline Long tour & 1.7900 & 0.1982 \\
\hline
\end{tabular}

Choice probabilities are also simulated over travel distance and accessibility to nearest bus stop and the same is given in Fig. 4 and Fig. 5. Choice probability of short tours has a steep negative slope up to $12 \mathrm{~km}$ travel distance and its value attains zero after that. Choice probability for medium tour increases has an increasing tendency up to $12 \mathrm{~km}$ and then it decreases. Long tour has an exponential increase in its choice probability with travel distance after $7 \mathrm{~km}$. After $17 \mathrm{~km}$ long tours have the highest probability to choose by the students. When the accessibility from nearest bus stop from place of residence is decreased short tour has a steep negative slope and both medium and long tour have positive slope. When the accessibility to nearest bus stop decreases beyond $2.6 \mathrm{~km}$, then the probability of choosing the long tours attains the highest value.

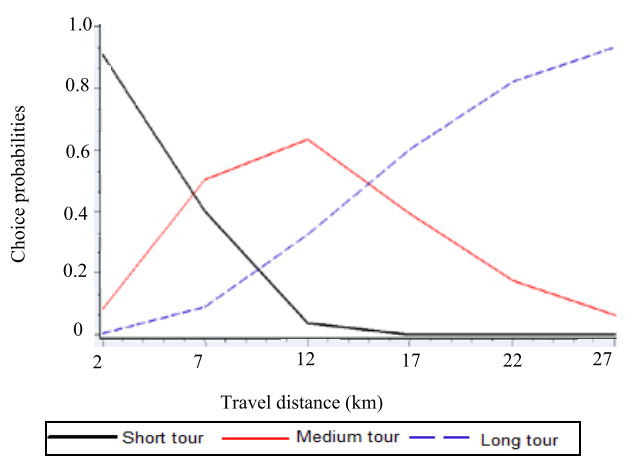

Fig. 4.

Simulation of Choice Probabilities of Total Travel Time Over Travel Distance 


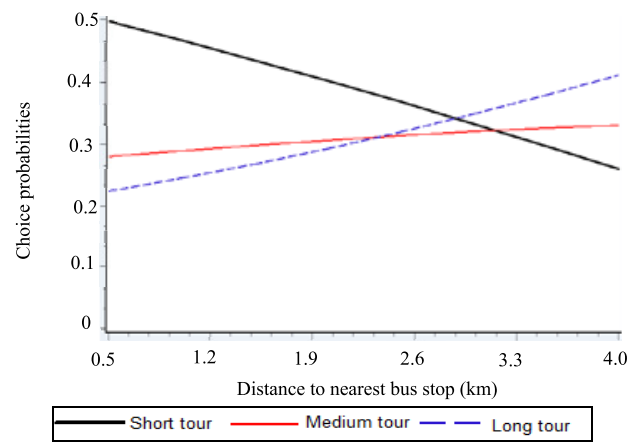

Fig. 5.

Simulation of choice probabilities of total travel time over Distance to nearest bus stop

\section{Conclusions}

In an ideal urban system the need to travel, in order to fulfill different personal and household needs for the dwellers, should be the minimum. Total travel time of a commuter reflects the extend up to which an individual need to travel for a particular activity, which is a least explored characteristics in transportation studies. Students always form a significant segment of the total population which is neglected in most of the transportation studies. Here total travel time model for students is calibrated to find out the influencing variables. Parameter estimates of total travel time model for students indicates the significant variables that influence total travel time of students for education are travel distance, gender, mode choice, population density of place of residence and also its accessibility to nearest bus stop. The goodness of fit measures and validation of the model indicates that the model is reasonably good for total travel time behaviour prediction. From point elasticities it is found that travel distance is relatively elastic over total travel time. Simulation of choice probabilities shows the variation in choice probabilities with respect to both travel distance and accessibility to public transport. Short tours have maximum probability for travel distance up to $7 \mathrm{~km}$, medium tours are more preferred from 7 to $15 \mathrm{~km}$ and after that long tours have maximum probability.

This paper provides valuable insights on total travel time behaviour of students in metropolitan region of a developing country. The findings from the present study are very relevant for attaining the goals of Smart City Mission for Kochi city, as one of its main focus is the improvement of transportation system in the city area by reducing in travel time, for an overall reduction in road congestion and improvement of air quality parameters (Smart city challenge Stage 2, Smart city proposal, 2016). Similar total travel time models can be developed for other segments of population like workers, nonworkers etc., to get the total picture about the whole population and post estimation analysis of the calibrated model can be done to identify the policy variables which have the potential to reduce the total travel time for different activities. 


\section{Acknowledgements}

The authors gratefully acknowledge the financial support received for the research from Woman Scientist Division, Kerala State Council for Science, Technology \& Environment (KSCSTE), Government of Kerala, India.

\section{References}

Ashalatha, R.; Manju, V.S.; Zacharia, A. B. 2013. Mode Choice Behavior of Commuters in Thiruvananthapuram City, Journal of Transportation Engineering 139(5): 494-502. doi: 10.1061/(ASCE)TE.1943-5436.0000533.

Chapin, F.S. 1974. Human activity patterns in the city: Things people do in time and in space, Wiley-Interscience 13.

Cullen, I.; Godson, V. 1975. Urban networks: the structure of activity patterns, Progress in planning 4: 1-96.

Frank, L.; Bradley, M.; Kavage, S.; Chapman, J.; Lawton, T.K. 2008. Urban form, travel time, and cost relationships with tour complexity and mode choice, Transportation 35(1): 37-54. doi: 10.1007/s11116-0079136-6.

Gopi, K.M.; Thanelimalil, S.G.; Anjaneyulu, M.V.L.R. 2018. Travel Survey Instrument Design Integrating Residential location Characteristics and Survey Administration for Kochi City, India, International Journal of Traffic and Transportation Engineering 8(2): 198-212.

Greene, W.H. 2016. NLOGIT Version 6.0, Reference Guide. Econometric Software, Inc.

Hägerstrand, T. 1970. What about people in Regional Science?, Papers in regional science 24(1): 6-21.
Hensher, D.A.; Rose, J.M.; Greene, W.H. 2005. Applied choice analysis a primer. Cambridge university press, New York.

Khattak, A.; Wang, X.; Son, S.; Agnello, P. 2011. Travel by university students in Virginia: Is this travel different from travel by the general population?, Transportation Research Record: Journal of the Transportation Research Board 2255: 137-145. doi: 10.3141/2255-16.

Koppelman, F.S.; Bhat, C. 2006. A self-instructing course in mode choice modeling: multinomial and nested logit models. U. S. Department of Transportation Federal Transit Administration, USA.

Lomax, T.; Schrank, D. 2010. Developing a Total Travel Time Performance Measure A Concept Paper. Texas Transportation Institute, USA.

Manoj, M.; Verma, A. 2015. Activity-travel behaviour of non-workers from Bangalore City in India, Transportation Research Part A 78: 400-424. doi: 10.1016/j. tra.2015.6.006.

Mitra, R.; Buliung, R.N. 2014. The influence of neighborhood environment and household travel interactions on school travel behavior: an exploration using geographically-weighted models, Journal of Transport Geography 36: 69-78. doi: 10.1016/j. trangeo.2014.03.002.

Müller, S.; Tscharaktschiew, S.; Haase, K. 2008. Travelto-school mode choice modelling and patterns of school choice in urban areas, Journal of Transport Geography 16(5): 342-357. doi: 10.1016/j.trangeo.2007.12.004.

Parthan, K.; Srinivasan, K.K. 2013. Investigation of Alternate Behavioural Frameworks for Mode Choice Decisions of Workers in Chennai City, Procedia-Social and Behavioral Sciences 104: 573-582. doi: 10.1016/j. sbspro.2013.11.151. 
Salonen, M.; Toivonen, T. 2013. Modelling travel time in urban networks: comparable measures for private car and public transport, Journal of Transport Geography 31: 143-153. doi: 10.1016/j.jtrangeo.2013.06.011.

Smart city challenge Stage 2, Smart city proposal. 2016. Ministry of Urban development, Government of India.

Sreela, P.K.; Anjaneyulu, M.V.L.R. 2018. Modelling the activity travel pattern of commuters in a medium sized city in India, International Journal for Traffic and Transport Engineering 8(4): 481 - 493. doi: 10.7708/ ijtte.2018.8(4).07.
Van Exel, N.J.A.; Rietveld, P. 2010. Perceptions of public transport travel time and their effect on choicesets among car drivers, Journal of Transport and Land Use 2(3/4): 75-86.

Whalen, K.E.; Páez, A.; Carrasco, J. A. 2013. Mode choice of university students commuting to school and the role of active travel, Journal of Transport Geography 31: 132-142. doi: 10.1016/j.trangeo.2013.06.008. 